\title{
Microporous Pillared Paddle-Wheel Frameworks based on Mixed Ligand Coordination of Zinc Ions
}

\author{
Bao-Qing Ma, Karen L. Mulfort and Joseph T. Hupp* \\ Contribution from the Department of Chemistry, Center for Nanofabrication and \\ Molecular Self-Assembly, and Institute for Environmental Catalysis, Northwestern \\ University, 2145 Sheridan Road, Evanston, Illinois 60208.
}

\section{Supporting Materials}

* To whom correspondence should be addressed. E-mail: j-hupp@ northwestern.edu (J. T. Hupp). 


\section{Synthesis}

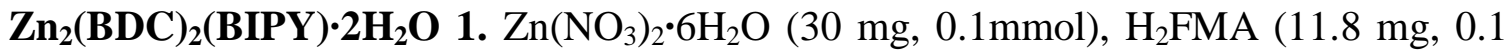
$\mathrm{mmol})$ and BIPY (8 $\mathrm{mg}, 0.05 \mathrm{mmol})$ were mixed in a mixture solution containing $10 \mathrm{~mL}$ DMF, $1 \mathrm{~mL}$ ethanol and $1 \mathrm{~mL}$ water in a small vial, which was capped and heated to 60 ${ }^{\circ} \mathrm{C}$ in an oil bath for $2 \mathrm{~d}$, and then cooled to room-temperature. Colorless crystals of the product were formed and collected by filtration and washed with DMF several times. The product loses solvents in air and insoluble in water and common organic solvents such as ethanol, acetonitrile, acetone, chloroform, and DMF.

$\mathbf{Z n}_{\mathbf{2}}$ (BDC) $)_{2}$ (BIPY) $\cdot \mathbf{n D M F}$ 2. $\mathrm{Zn}\left(\mathrm{NO}_{3}\right)_{2} \cdot 6 \mathrm{H}_{2} \mathrm{O}(30 \mathrm{mg}, 0.1 \mathrm{mmol}), \mathrm{H}_{2} \mathrm{BDC}(16.6 \mathrm{mg}, 0.1$ $\mathrm{mmol})$ and BIPY ( $8 \mathrm{mg}, 0.05 \mathrm{mmol})$ were mixed with $10 \mathrm{~mL}$ DMF solution in a small vial, which was capped and heated to $80{ }^{\circ} \mathrm{C}$ in an oil bath for $2 \mathrm{~d}$, and then cooled to room-temperature. Colorless crystals of the product were formed and collected by filtration and washed with DMF several times. The product loses solvents in air and insoluble in water and common organic solvents such as ethanol, acetonitrile, acetone, chloroform, and DMF.

$\mathbf{Z n}_{2}$ (NDC) $)_{2}$ (BIPY) $\cdot$ nDMF 3. $\mathrm{Zn}\left(\mathrm{NO}_{3}\right)_{2} \cdot 6 \mathrm{H}_{2} \mathrm{O}(30 \mathrm{mg}, 0.1 \mathrm{mmol}), \mathrm{H}_{2} \mathrm{NDC}(21.6 \mathrm{mg}, 0.1$ $\mathrm{mmol})$ and BIPY ( $8 \mathrm{mg}, 0.05 \mathrm{mmol})$ were mixed with $10 \mathrm{~mL}$ DMF solution in a small vial, which was capped and heated to $80{ }^{\circ} \mathrm{C}$ in an oil bath for $2 \mathrm{~d}$, and then cooled to room-temperature. Colorless crystals of the product were formed and collected by filtration and washed with DMF several times. The product loses solvents in air and insoluble in water and common organic solvents such as ethanol, acetonitrile, acetone, chloroform, and DMF.

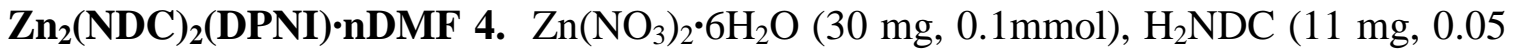
$\mathrm{mmol}$ ) and DPNI (11 mg, $0.025 \mathrm{mmol})$ were mixed with $10 \mathrm{~mL}$ DMF solution in a small vial, which was capped and heated to $100{ }^{\circ} \mathrm{C}$ in an oil bath for $2 \mathrm{~d}$, and then cooled to room-temperature. Yellow crystals of the product were formed and collected by filtration and washed with DMF several times. The product loses solvents in air and insoluble in water and common organic solvents such as ethanol, acetonitrile, acetone, chloroform, and DMF.

$\mathbf{Z n}_{2}$ (BPDC) $)_{2}$ (DPNI) $\cdot \mathbf{n D M F}$ 5. $\mathrm{Zn}\left(\mathrm{NO}_{3}\right)_{2} \cdot 6 \mathrm{H}_{2} \mathrm{O}$ (30 mg, 0.1 mmol), $\mathrm{H}_{2} \mathrm{BPDC}(24 \mathrm{mg}, 0.1$ $\mathrm{mmol})$ and DPNI (21 mg, $0.05 \mathrm{mmol})$ were mixed with $10 \mathrm{~mL}$ DMF solution in a small vial, which was capped and heated to $80{ }^{\circ} \mathrm{C}$ in an oil bath for $2 \mathrm{~d}$, and then cooled to room-temperature. Yellow crystals of the product were formed and collected by filtration and washed with DMF several times. The product loses solvents in air and insoluble in water and common organic solvents such as ethanol, acetonitrile, acetone, chloroform, and DMF. 


\section{Crystal structure determination:}

Single crystals were mounted on a BRUKERS SMART CCD 1000 diffractometer equipped with a graphite-monochromated $\operatorname{MoKa}(1(\lambda=0.71073 \AA)$ radiation source in a cold nitrogen stream. For 1, diffraction data were collected for both as-synthesized and evacuated crystals. The disordered DMF can be reasonably modeled in $\mathbf{1}$. For 2-5, contributions from disordered solvent molecules were removed by the SQUEEZE routine (PLATON), and the outputs from the SQUEEZE calculations are attached to each CIF file. All crystallographic data were corrected for Lorentz and polarization effects (SAINT), and face-index absorption corrections. The structures were solved by direct methods and refined by the full-matrix least-squares method on F2 with appropriate software implemented in the SHELXTL program package. All the non-hydrogen atoms except for those of disordered solvent molecules in $\mathbf{1}$ were refined anisotropically. Hydrogen atoms were added at their geometrically ideal positions. One benzene dicarboxylic acid and one of pyridine rings of 4,4'-BIPY in $\mathbf{2}$ are disordered over two positions. The 4,4'-BIPY and one of phenyl rings of 2,6-naphthalenedicarboxylic acid in $\mathbf{3}$ are disordered over two positions. 


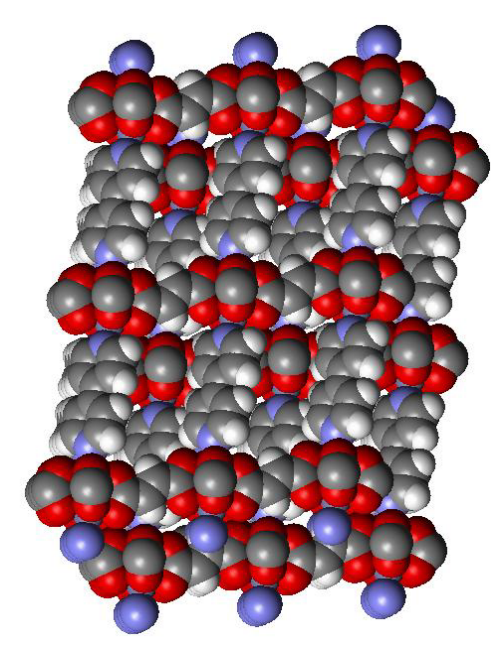

a

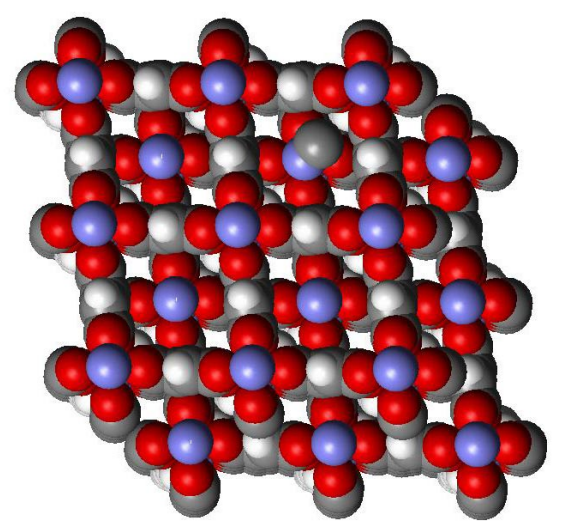

b

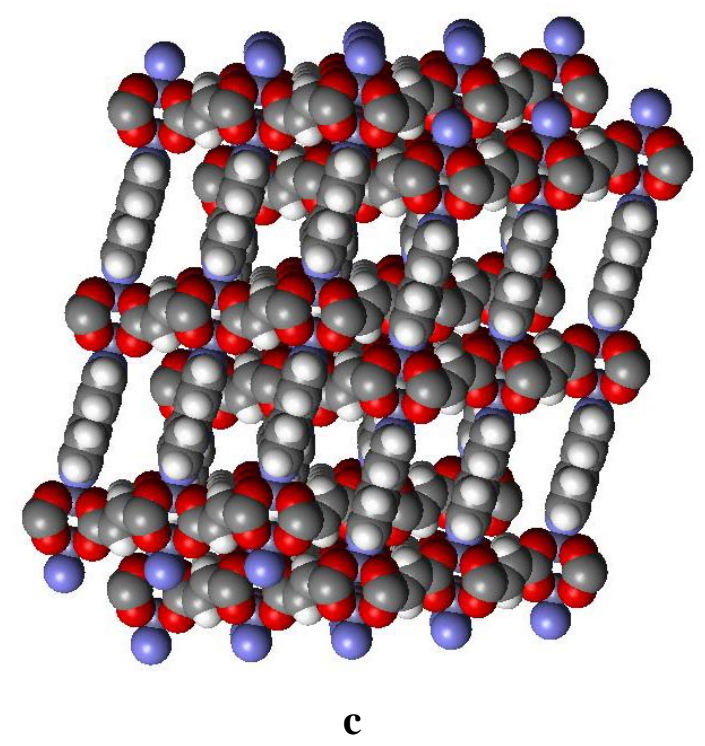

Figure S1. Space filling diagram of compound 1 along different directions, showing the two-fold interpenetration and remaining channels. 


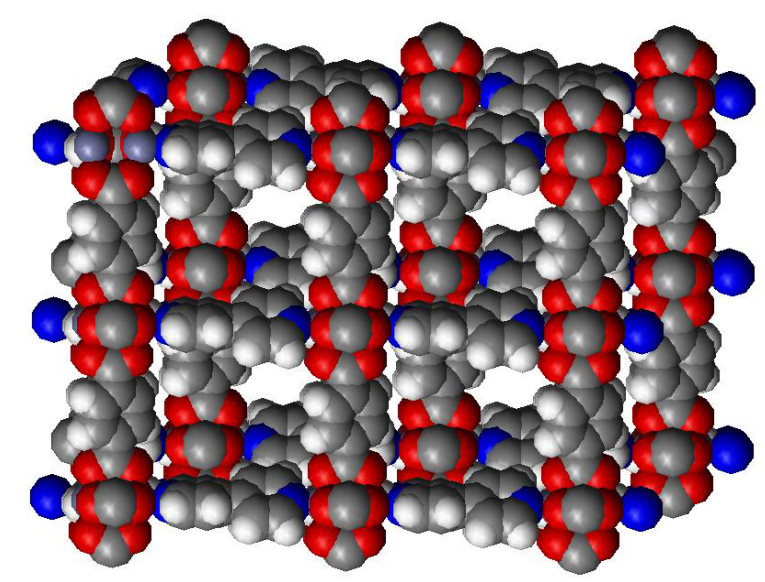

a

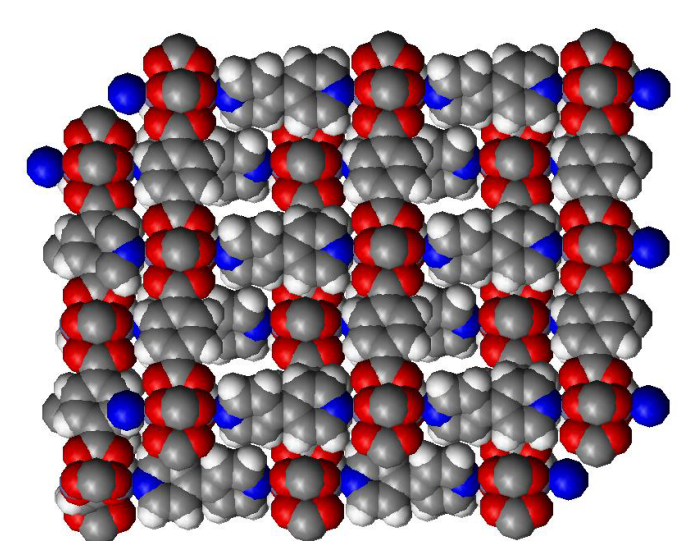

b

Figure S2. Space filling diagram of compound 2 along $a$ (a) and $b$ (b) directions, showing the two-fold interpenetration and remaining channels. 


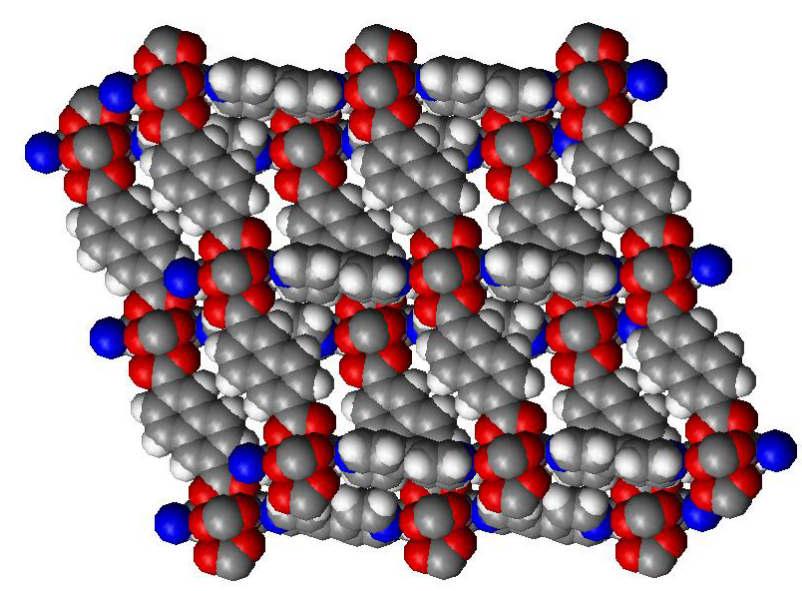

a

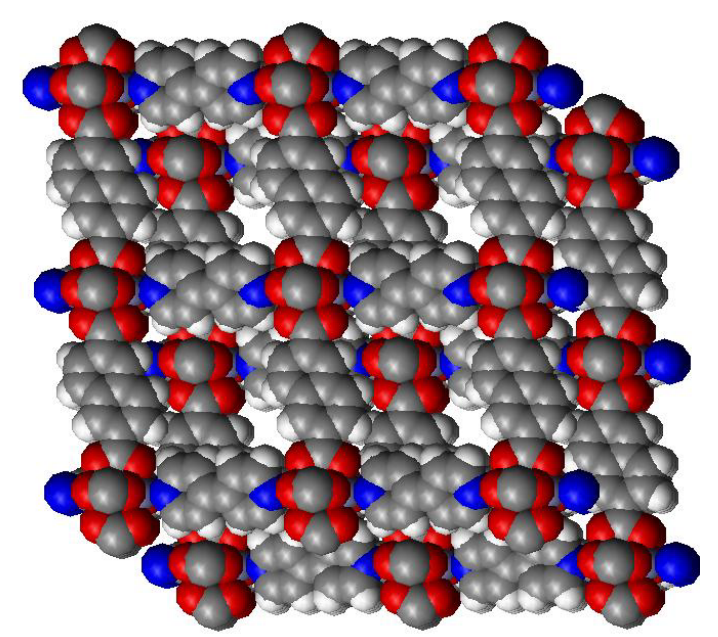

b

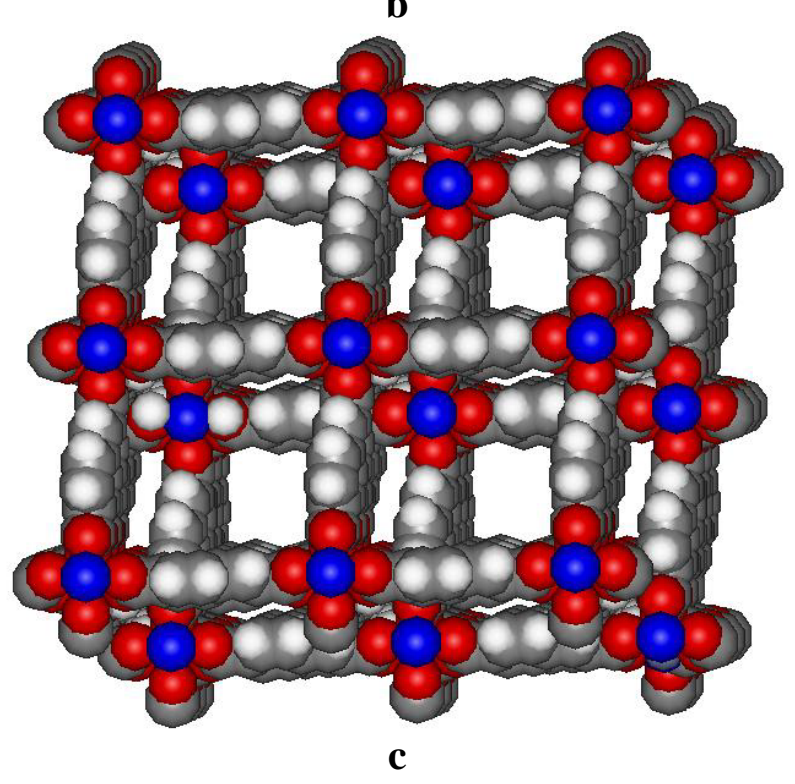

Figure S3. Space filling diagram of compound $\mathbf{3}$ along $a(\mathbf{a}), b(\mathbf{b})$ and $c$ (c) directions, showing the two-fold interpenetration and remaining channels. 


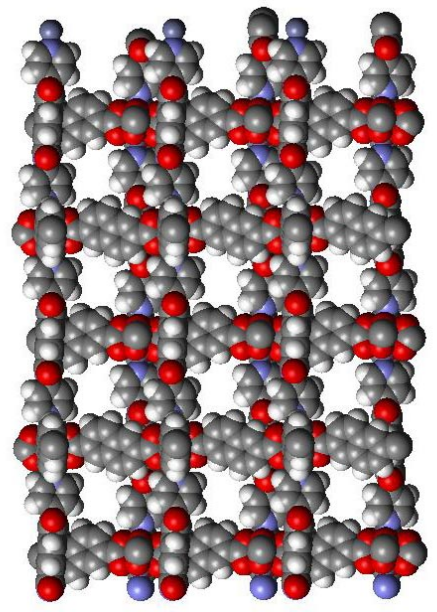

a

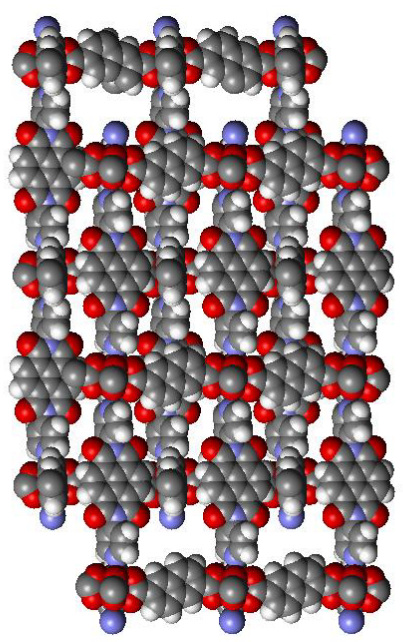

b

Figure S4. Space filling diagram of compound 4 along $a(\mathbf{a})$ and $c$ (b) directions, showing the two-fold interpenetration and remaining channels. 

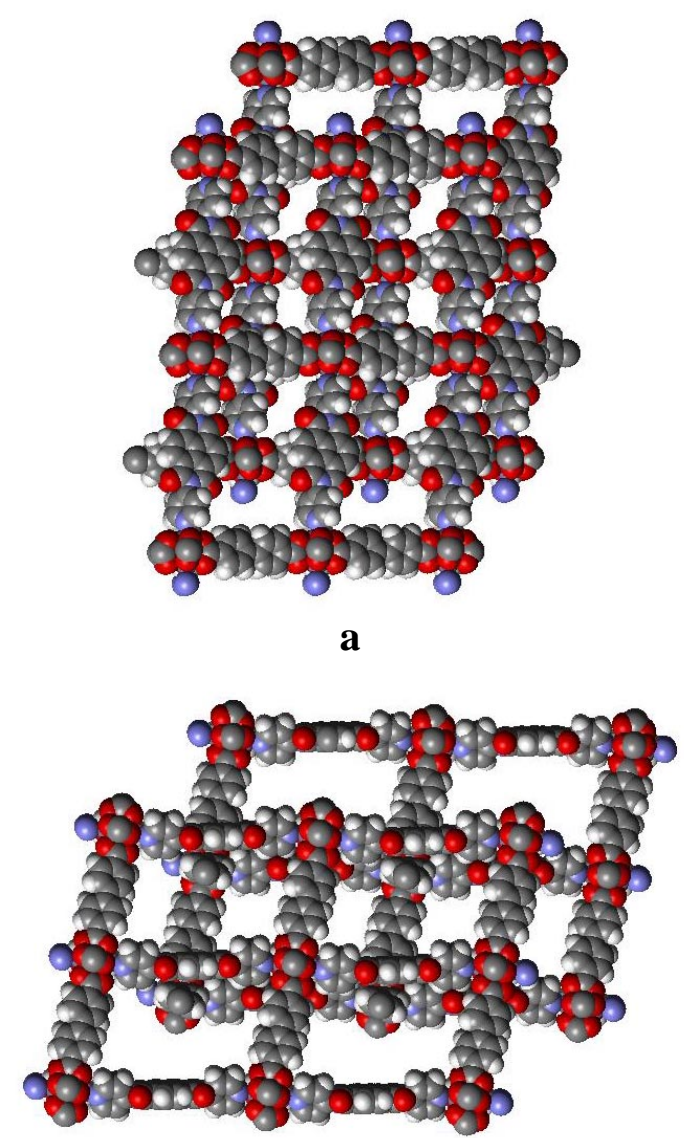

b

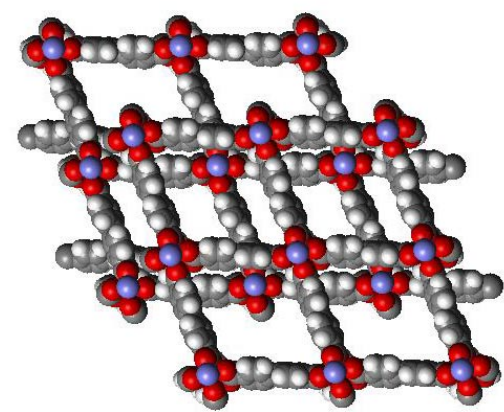

c

Figure S5. Space filling diagram of compound 5 along $a(\mathbf{a}), b(\mathbf{b})$ and $c$ (c) directions, showing the two-fold interpenetration and remaining channels. 


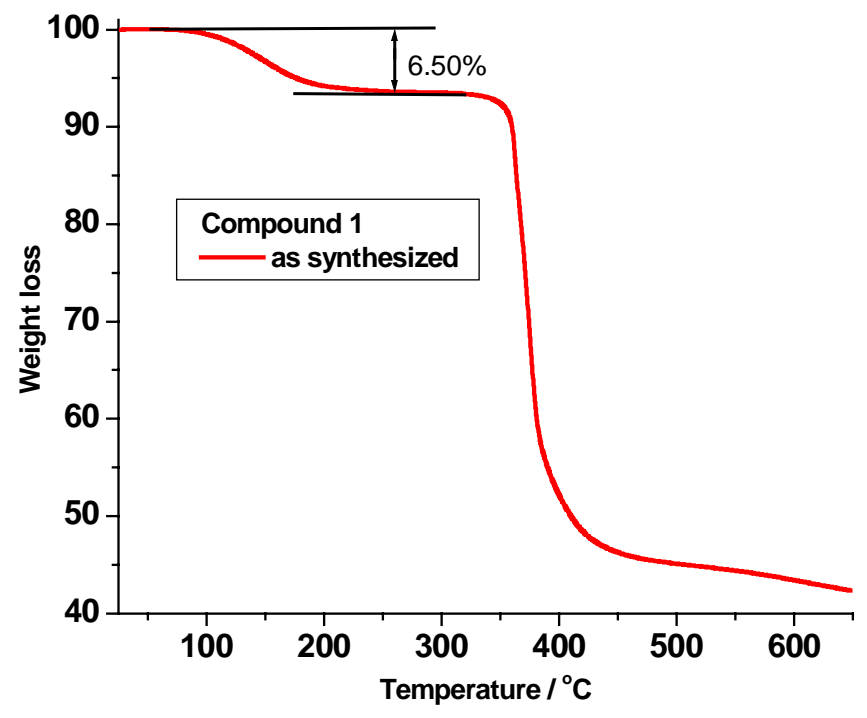

a

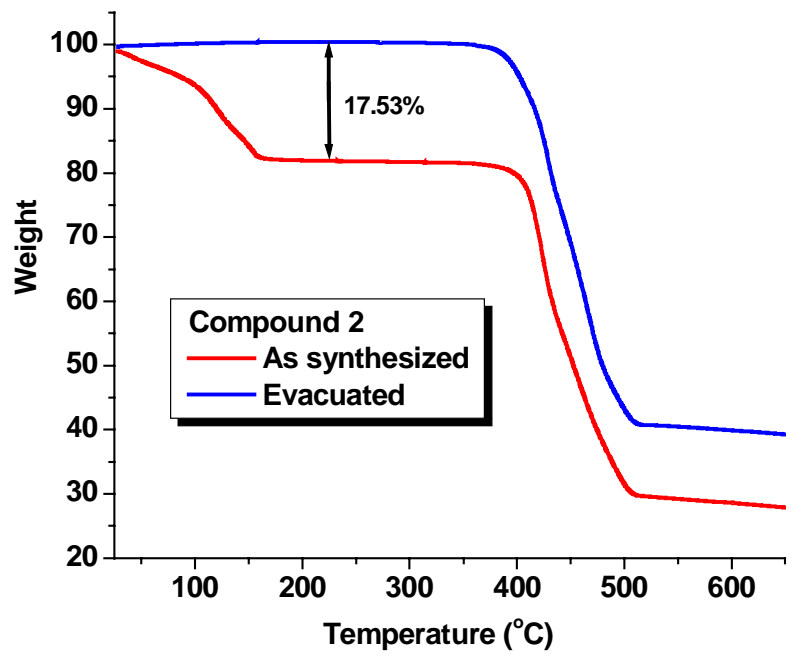

b 


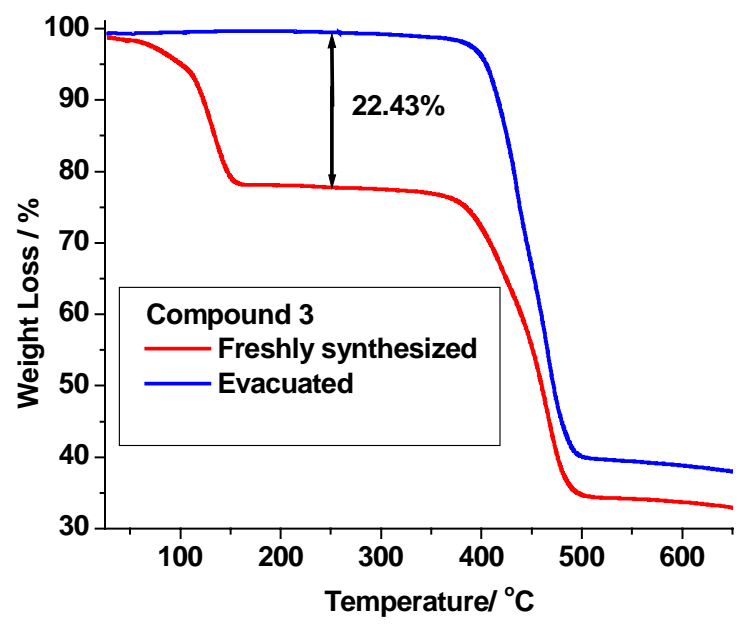

c

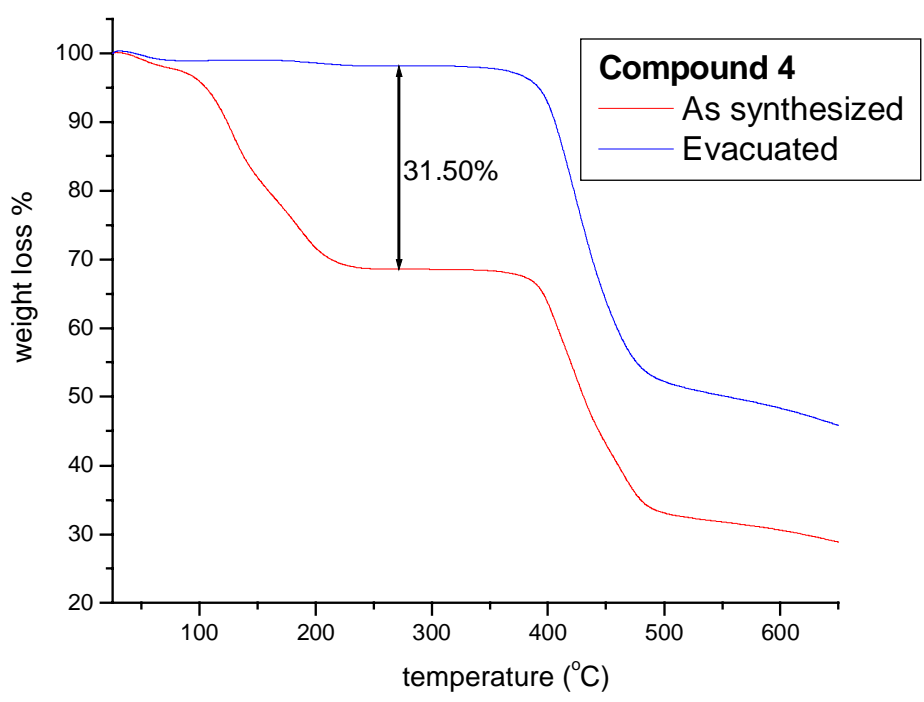

d

Figure S6. TGA for the freshly synthesized and evacuated compounds 1 (a), 2 (b), 3 (c) and 4 (d). 


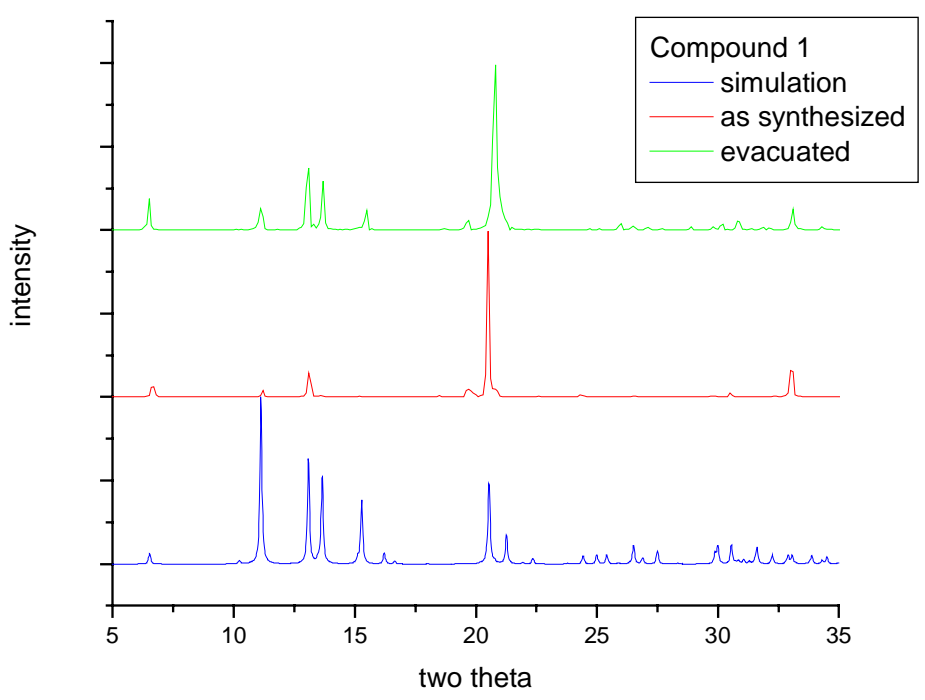

a

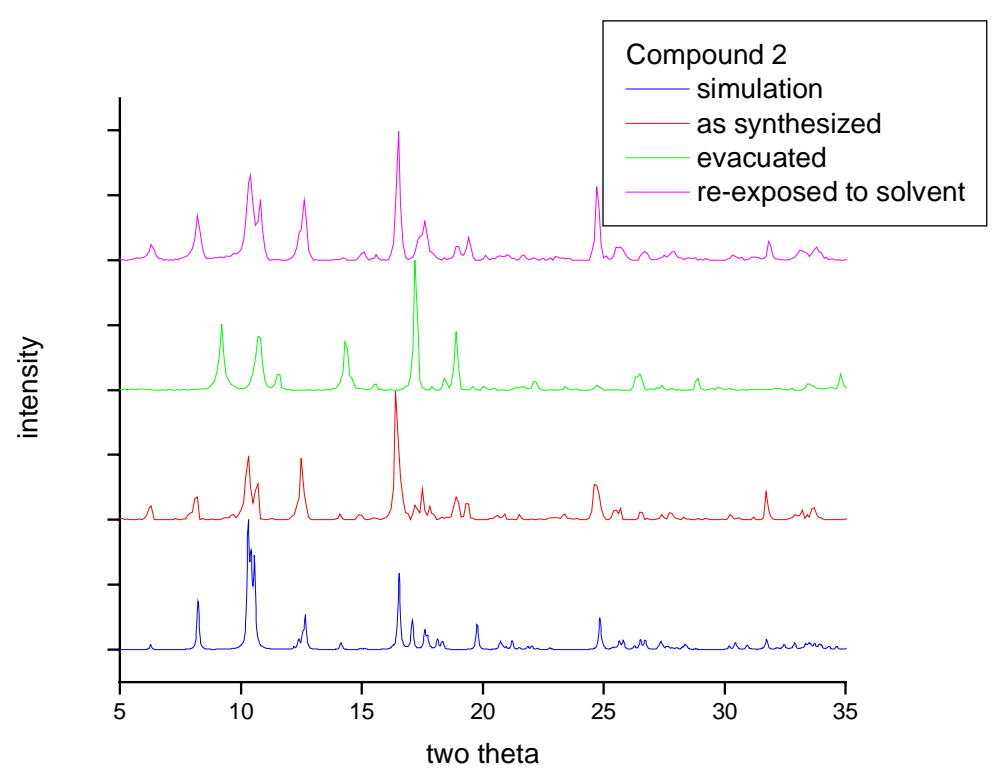

b 


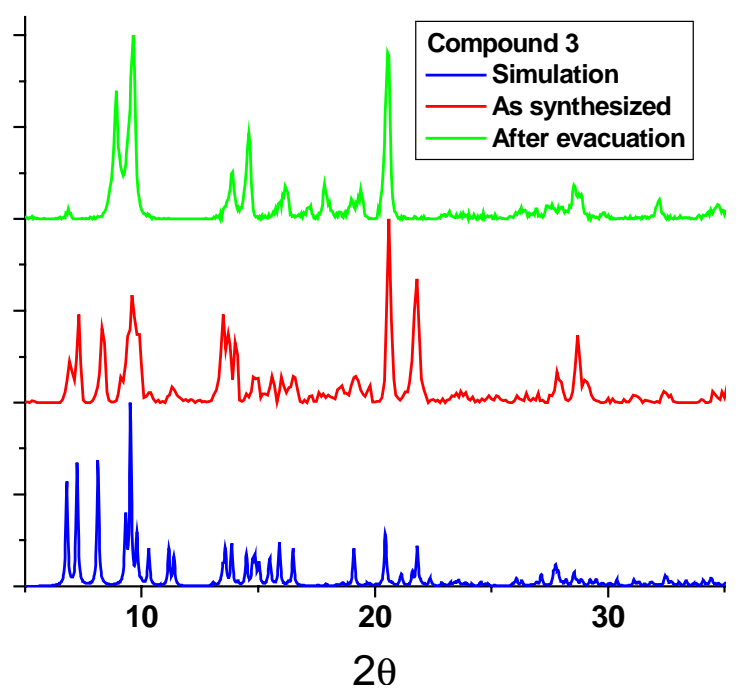

c

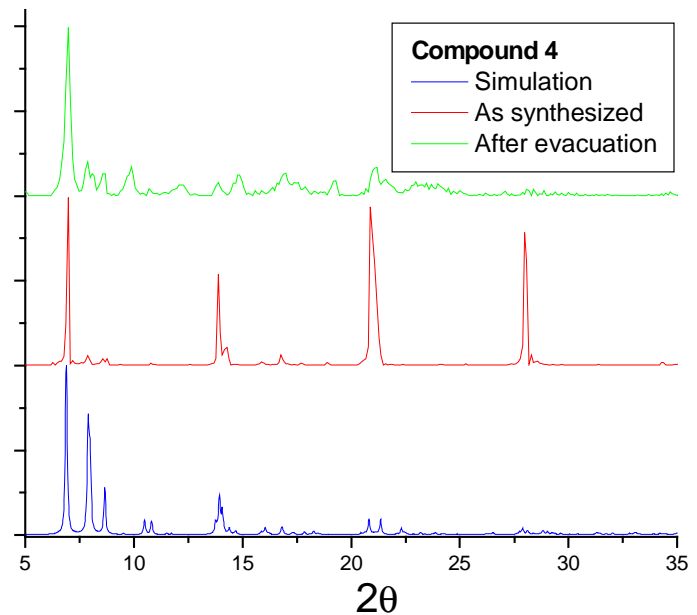

d 


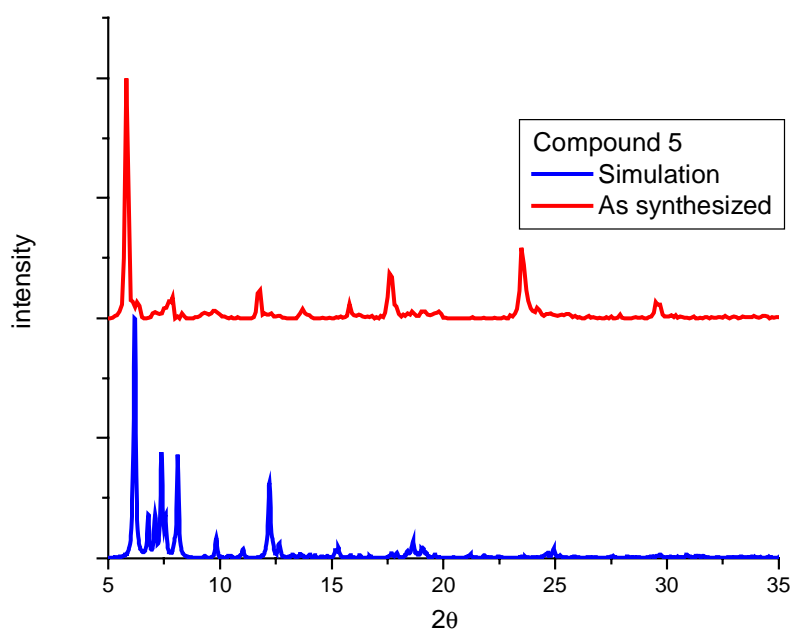

e

Figure S7. XRD pattern for the compounds 1 (a), 2 (b), 3 (c), 4 (d) and $\mathbf{5}(\mathrm{e})$. 